\title{
Stroke Care in an Australian Rural Private Health Care Setting
}

\author{
Nirosen Vijiaratnam1,2*, Bernard Yan³, Pamela Anjara², Thomas Kraemer1, Mandy Lau', \\ Brett Knight ${ }^{1}$ \\ ${ }^{1}$ St. John of God Hospital, Ballarat, Australia \\ ${ }^{2}$ Western Health, Melbourne, Australia \\ ${ }^{3}$ Melbourne Health, Melbourne, Australia \\ Email: "Nirosen.vijiaratnam@wh.org.au,
}

Received 22 November 2014; revised 15 December 2014; accepted 21 December 2014

Academic Editor: Yujiao Jennifer Sun, University of California, USA

Copyright (C) 2015 by authors and Scientific Research Publishing Inc.

This work is licensed under the Creative Commons Attribution International License (CC BY).

http://creativecommons.org/licenses/by/4.0/

(c) (i) Open Access

\begin{abstract}
Stroke is a leading cause of disability and death in Australia. There is a clear benefit in caring for stroke patients in stroke care units. Access to these centres is limited particularly in the rural setting. Certified stroke care units in the private health care setting are also unheard of. The superiority of these units is thought to be due to better adherence to processes of care (early utility of CT scan, allied health input within 24 hours, neurological observations, DVT prophylaxis and appropriate use of antiplatelet and anticoagulant use). We audited care of 100 patients who presented to the St. John of God Hospital (rural private hospital) over a period of 3 years. This included baseline demographics, adherence of processes of care, utility of appropriate investigations, and outcome measures such as discharge destination, level of function at discharge and complication rates. These data were compared with the national stroke report (AuSCR) and adherence to processes of care was compared with the SCOPE study (the first study to establish the benefit of POC). When compared with data from the AuSCR national report 2012, we found a higher mortality rate, an increased rate of disability on discharge, and a mixed adherence to processes of care. We also found a significant proportion of patients $(\mathbf{4 0 \% )}$ ) who were eligible to receive thrombolysis but did not. Overall we found that there were significant strengths to be drawn upon in the rural private healthcare setting and a more organised approach could improve outcomes.
\end{abstract}

\section{Keywords}

Stroke, Outcomes, Processes of Care, Stroke Care Units, Rural Private Healthcare

\footnotetext{
${ }^{*}$ Corresponding author.
} 


\section{Introduction}

Stroke is the leading cause of permanent disability [1] and second most common cause of death in Australia [2]. Ischemic strokes comprise about $80 \%$ of all strokes with the remaining being intracranial haemorrhages.

Stroke care units (SCU) have been shown to improve survival and independence when compared with general ward management [3]. Access to this specialised service has however been limited by resources. This is particularly the case in the rural setting. This may partially explain the poorer outcomes in stroke treatment seen in the rural setting [4] [5]. Divergence of evidence based stroke care pathways may also explain this difference in outcome [6]. In particular, lower adherence to key processes of care (POC) in conventional care wards in Australia is thought to be the explanation for poorer outcomes when compared to SCU [7]. The higher rate of thrombolysis in SCU is also thought to be a contributing factor [8].

Survival and functional outcome of strokes in the private healthcare setting is less well established. To our knowledge there are no published data from the Australian private healthcare setting. Analysis of prospective data from a stroke registry in Switzerland (access to stroke units with standardised treatment pathways was the same regardless of insurance status) found that patients with private health insurance presented with less severe strokes (NIHSS scores) and had a better outcome at 1 week and 3 months (mRS) [9].

We aim to perform a retrospective audit of stroke patients who presented to St. John of God (SJOG) from 2011-2014. Patient demographics including risk factors for stroke will be noted. Adherence to POC and outcomes on discharge will be noted. The data will be compared with the AuSCR national report 2012 and POC adherence compared to the SCOPE study [7]. We hypothesise that outcomes will be worse than the national average and that adherence to processes of care and utility of standard stroke investigations will be poorer.

\section{Methods}

We retrospectively collected data from 100 patients admitted with acute ischemic stroke between November 2011 and April 2014 at the St. John of God Hospital in Ballarat, Victoria. This is the only private hospital in the region and serves a catchment area of about 100,000 inhabitants. No patients presenting to this hospital received thrombolysis. Patients were under the care of a group of general physicians with occasional input from a neurologist. The hospital did not have obligatory POC. The only formal nursing obligation was a referral to allied health (physiotherapy, occupational therapy and speech therapy) on admission and allied health review should be performed within 24 hours. There was no formal stroke care pathway otherwise.

We collected demographic data (age, gender), type of stroke using the Oxfordshire classification and severity using the National Institute of Health Stroke Scale (NIHSS). The time of onset of symptoms to the time of admission to the SJOG emergency department and the NINDS criteria for thrombolysis were used to extrapolate eligibility for thrombolysis. POC adherence being analysed were similar to those previously shown as being important in the SCOPES study. Briefly, these were: 1) activities within 24 hours, which were CT scanning, swallow assessment, allied health assessment, and neurological observations; 2) documentation of the patients premorbid function and discharge needs; 3) management: measures to avoid aspiration, deep vein thrombosis prophylaxis and appropriate use of antiplatelet agents at discharge. Utility of appropriate investigations of stroke aetiology and risk factors (carotid Doppler study, ECG, telemetry, transthoracic echocardiography, fasting lipids and blood glucose) was also recorded. Outcome measurement collected included the modified rankin score (mRS) on discharge and length of stay. A mRS of 0 - 2 is considered a favourable outcome. This audit was approved by the local ethics committee.

\section{Results}

A hundred admissions from November 2011 to30 April 2014 were included in this audit. This was an average of 3.33 admissions per month. Table 1 shows the baseline demographic characteristics (age, gender, risk factors, stroke type and average severity). The mean age was 77 (standard deviation (SD) 10) and about 70\% were females. Majority of patients were independent (94\%) prior to admission. Approximately $50 \%$ of patients suffered a more disabling total anterior circulation infarct (TACI). The average NIHSS score on admission was 7.3 (SD 4.4).

Adherence to POC was variable across indicators (Table 2). All patients received CT scanning and allied health input (physiotherapy, occupational therapy and speech therapy) within 24 hours. It was not part of hospital 
Table 1. Patient characteristics.

\begin{tabular}{ccc}
\hline Baseline demographics & St. John of God (SJOG) & AuSCR \\
\hline Age, $\mathbf{y}$ & & $74(14)$ \\
Mean (SD) & $77.6(10.6)$ & $48 \%$ \\
Female & $69(69 \%)$ & N/A \\
Independent before stroke (mRS 0 - 2) & $94(94 \%)$ & N/A \\
Risk factors & & \\
Smoker & $18(18 \%)$ & \\
Atrial fibrillation & $34(34 \%)$ & \\
Hypertension & $73(73 \%)$ & \\
Diabetes & $27(27 \%)$ & \\
Hypercholesterolemia & $36(36 \%)$ & N/A \\
TIA & $4(4 \%)$ & N/A \\
Previous stroke & $22(22 \%)$ & \\
TACI & & \\
PACI & $56(56 \%)$ & $5(5 \%)$ \\
LACI & $31(31 \%)$ & \\
POCI & $7.3 \pm 4.4$ & \\
NIHSS at admission (average \pm SD) & & \\
\hline
\end{tabular}

Table 2. Processes of care (POC).

\begin{tabular}{ccc}
\hline Process indicator & Adherence to processes of care POC (\%) & Scopes \\
\hline CT scan $<$ 24 h since admission & St. John of God & $96 \%$ \\
Dysphagia screen $<24$ h since admission & 100 & $56 \%$ \\
Speech pathologist within $\mathbf{2 4} \mathbf{h}$ & 0 & $39 \%$ \\
Allied health $<\mathbf{2 4}$ h since admission & 100 & $61 \%$ \\
Neurological observations & 100 & $73 \%$ \\
Documented premorbid function & 0 & $97 \%$ \\
Documented discharge needs & 100 & $95 \%$ \\
DVT prophylaxis & 100 & $59 \%$ \\
Measures to avoid aspiration & 63 & $94 \%$ \\
Discharged on appropriate antiplatelet & 100 & $64 \%$ \\
agent/anticoagulation & 97 & $12 \%$ (national thrombolysis average) \\
\hline
\end{tabular}

policy that all patients should receive a swallow test on admission or ongoing neurological observations as a routine for the first 24 hours. Measures to avoid aspiration were in place and adhered to in all cases where instructions were given post speech therapist review. Deep venous thrombosis prophylaxis was only instituted in $63 \%$ of cases required (not on anticoagulation and immobile). $40 \%$ of patients would have qualified for thrombolysis under NINDS criteria.

The utility of investigation was highly variable (Table 3). $57 \%$ of patients received a MRI scan. Carotid Dop- 
pler studies were only done in 57\% of patients. Whilst all patients had an ECG at baseline only $23 \%$ of patients were monitored for at least 24 hours. The utility of tests for risk factor stratification (fasting blood glucose and lipids) was also low (15\%).

The average length of stay was 11.9 days (Table 4). 55\% of patients were discharged home independent. 30\% of patients were discharged with significant disability to care. Mortality in this centre was $15 \% .42 \%$ of the remaining patients were discharged to rehabilitation with $42.4 \%$ discharged directly home. $9.4 \%$ required high level care nursing home support (Table 5).

Only 1 patient developed haemorrhagic transformation in our cohort. The most common complication during admission was a urinary tract infection (UTI). This was followed by 5 patients developing pneumonia. No patients developed any form of venous thrombosis.

Table 3. Investigations.

\begin{tabular}{cc}
\hline Investigation & Percentage received (\%) \\
\hline ECG & 100 \\
Telemetry (24 h) & 23 \\
Trans thoracic echocardiogram & 37 \\
Carotid Doppler & 57 \\
MRI & 57 \\
Fasting lipids & 15 \\
Fasting blood glucose/HbA1c & 15 \\
\hline
\end{tabular}

Table 4. Outcomes.

\begin{tabular}{|ccc|}
\hline & St. John of God & AuSCR \\
\hline $\begin{array}{c}\text { Length of stay (average } \pm \text { SD) } \\
\text { Length of stay (median) }\end{array}$ & $11.9 \pm 13.9$ & N/A \\
$\begin{array}{c}\text { Discharged to rehabilitation } \\
\text { (\% of surviving patients) }\end{array}$ & 7 & 5 \\
$\begin{array}{c}\text { Discharged home (\% of surviving patients) } \\
\text { Discharged to low level care } \\
\text { (\% of surviving patients) } \\
\text { Discharged to high level care } \\
\text { (\% of surviving patients) } \\
\text { Independence (mRS 0 - 2) }\end{array}$ & $36(42 \%)$ & $52 \%$ \\
Dependence (mRS 3 - 5) & $46(42.4 \%)$ & $1 \%$ \\
$\quad$ Mortality (mRS 6) & $8(9.4 \%)$ & $6 \%$ \\
\hline
\end{tabular}

Table 5. Complications.

\begin{tabular}{ccc} 
Complication & Total \\
\hline Haemorrhagic transformation & 1 \\
Pneumonia & 5 \\
DVT & 0 \\
PE & 0 \\
UTI & 10 \\
\hline
\end{tabular}




\section{Discussion}

Our data provides some imposing insights into the outcomes of patients presenting with ischemic stroke and managed in a rural private health care setting. The first point to be raised is that the unit has an average of 3.33 stroke admissions per month or about 36 per year. This correlates with a low to medium annual number using the preferred definition of the registry of the Canadian stroke network. This reflects the reality of patient volumes in this setting and probably provides a realistic presentation of outcomes in similar settings in Australia. Also about a third of the AuSCR data are supplied by hospitals of this size (11/31).

The patient demographics and risk factors are compatible with established stroke outcome studies [4] [5]. The female representation in this study is however high when compared to the AuSCR data. Interestingly the average NIHSS score of the study of 7.3 is similar to the Swiss study (mean NIHSS 7.8) which found that patients with private health insurance had a lower NIHSS score on admission. We could not make a comparison with the Australian average, as this was not provided in the report.

Overall, adherence to POC was variable when compared to the SCOPES study [7]. All patients received CT scanning on presentation and allied health input within 24 hours as part of hospital protocol. This was exceptional in comparison to a CT adherence rate of $96 \%$ and allied health review rate of $61 \%$ in the SCOPE study. Dysphagia screening was not part of standard care. All patients however received a speech therapist review within 24 hours, which is a superior assessment. DVT prophylaxis rates at 63\% compares to a rate $59 \%$ in the SCOPES study. Having not adhered to neurological observations would most certainly have impacted on outcomes with the SCOPES study finding that not adhering to one POC increasing mortality by 3.09 [7]. Overall, this would fall into the thorough adherence category in the SCOPES study, which is encouraging. This group was found to be more likely to be discharged home and be independent at 28 weeks in that study. What is also important to note is that only 34\% of SCUs in that study achieved this level of adherence [7]. There still remains room for improving on adherence to processes of care to mimic SCU. There is evidence of trying to establish this in a rural setting in Australia making significant difference in outcomes [10].

The low rate of adherence to evidence based investigations is of particular concern and is likely to have contributed to outcomes. Only $23 \%$ of patients received telemetry and $15 \%$ of patients were investigated with fasting bloods. National adherence rates are not available for comparison. It is important to note however that patients in SCUs in the SCOPES study were more likely to receive these investigations [7]. The question on whether these investigations contributed to better outcomes is not clear.

The median length of stay of 7 days was high (Table 4). This compares with a median stay of 4 days in non-stroke units and 5 days in stroke units in the 2012 AuSCR report [9]. The percentage of patients discharged home of $42.4 \%$ was low when compared to the national average of $52 \%$ [9]. The percentage discharged to rehabilitation of $42 \%$ was high compared with the AuSCR average of $27 \%$. The percentage requiring HLC in our group of $9.4 \%$ compares to the national average of $6 \%$. Our mortality of $15 \%$ compares to a national average of $12 \%$ [9]. It is rather subtle to state $42.4 \%$ is significantly lower than $52 \%$ while leaving $42 \%$ vs $27 \%$ or $9.4 \%$ vs 6\% comparable. Please use proper statistical analysis and present it in the method section and mark the significance level in the table (no comparator group so unable to perform stats. Can't do this against the national data as they only provide summary's).

There are a number of limitations to the generalization of this data. Firstly, it is from a single site and therefore may not reflect the overall performance of country private hospitals. The total number of 100 also makes it difficult to draw strong conclusions. Comparing our data with national statistics from hospitals that use thrombolysis also makes it difficult to draw conclusions regarding the degree to which other measured factors (POC, evidence based investigations) are contributing to outcome. Also the lack of a comparator group to assess statistical differences in outcome affects our ability to draw concrete conclusions. There are no other regional private hospitals and we did not have data from the adjacent public hospital to make a comparison. It is difficult there fore to perform any form of statistical comparison which would be most useful.

Regardless of these limitations, this audit provides us with multiple avenues for improvement in stroke care. Firstly, it is concerning that almost $40 \%$ of patients with the potential to receive thrombolysis did not receive the treatment. This is weighed against an average thrombolysis rate of $12 \%$ in the most recent AuSCR-annual report [9]. Thrombolysis was classed level 1a treatment within 3 hours and 1b treatment within 3 to 4.5 hours of stroke onset by the most recent American Stroke Society guidelines [11]. Whilst there is a lack of specialist input, a possible method of providing this treatment is with the utility of expertise from a telestroke network. This mo- 
dality has been used to facilitate the delivery of thrombolysis in multiple centres globally with equivalent safety outcomes to in person assessments [12]. The lack of neurological observations and the lack of utility of important investigations can be overcome with a streamlined stroke pathway that is typically utilised in stroke care units.

\section{Conclusion}

Whilst this was a small study in one centre, it provides some alerting insights into the reality of stroke care in both the rural setting and private healthcare setting. The poorer outcomes are most likely explained by the lack of utility of important investigations and the lack of a thrombolysis service. Adherence rates to POC are also likely to have a contributed to our outcomes. Future studies should involve reassessing outcomes after the institution of the above measures. Comparison of outcomes across the St. John's network of private hospital would also provide more. The challenge remains on how best to provide stroke care to patients outside publicly funded specialist tertiary centres. This study provides some insights into future directions.

\section{References}

[1] Senes, S. (2006) How We Manage Stroke in Australia. AIHW Cat. No. CVD 31. Australian Institute of Health and Welfare, Canberra.

[2] (2008) Australian Bureau of Statistics. 3303.0 Causes of Death 2206: Australia. Report No. 3303.0, Canberra.

[3] Stroke Unit Trialists' Collaboration (2003) Organised Inpatient (Stroke Unit) Care for Stroke (Cochrane Review). Oxford: Update Software. Cochrane Database of Systematic Reviews, 2003, Article ID: CD000197.

[4] Joubert, J., Prentice, L.F., Moulin, T., et al. (2008) Stroke in Rural Areas and Small Communities. Stroke, 39, 19201928. http://dx.doi.org/10.1161/STROKEAHA.107.501643

[5] Cadilhac, D.A., Kilkenny, M.F., Longworth, M., et al. (2011) Metropolitan-Rural Devide for Stroke Outcomes: Do Stroke Units Make a Difference? Internal Medicine Journal, 41, 321-326. http://dx.doi.org/10.1111/j.1445-5994.2010.02280.x

[6] Read, S.J. and Levy, J. (2005) Differences in Stroke Care Practices between Regional and Metropolitan Hospitals. Internal Medicine Journal, 35, 447-450.

[7] Cadilhac, D., Ibrahim, J., Pearce, D., et al. (2004) Multicentre Comparison of Processes of Care between Stroke Units and Conventional Care Wards in Australia. Stroke, 35, 1035-1040. http://dx.doi.org/10.1161/01.STR.0000125709.17337.5d

[8] Cadilhac, D., Lannin, N., Anderson, C., et al. (2013) The Australian Stroke Clinical Registry Annual Report 2012. The Florey Institute of Neuroscience and Mental Health, Report No. 4, 52.

[9] Rey, V., Faouzi, M., Huchmand-Zadeh, M., et al. (2011) Stroke Initial Severity and Outcome Relative to Insurance Status in a Universal Health Care System in Switzerland. European Journal of Neurology, 18, 1094-1097. http://dx.doi.org/10.1111/j.1468-1331.2010.03264.x

[10] Mccann, L., Groot, P., Charnley, C., et al. (2009) Excellence in Regional Stroke Care: An Evaluation of the Implementation of a Stroke Care Unit in Regional Australia. Australian Journal of Rural Health, 17, 273-278. http://dx.doi.org/10.1111/j.1440-1584.2009.01098.x

[11] Jaech, E., Saver, J., Adams, H., et al. (2013) Guidelines for the Early Management of Patients with Acute Ichemic Stroke: A Guideline for Healthcare Professionals from the American Heart Association/American Stroke Association. Stroke, 44, 870-947. http://dx.doi.org/10.1161/STR.0b013e318284056a

[12] Zaidi, S., Jumma, M., Urra, X., et al. (2011) Telestroke-Guided Intravenous Tissue-Type Plasminogen Activator Treatment Achieves a Similar Clinical Outcome as Thrombolysis at a Comprehensive Stroke Center. Stroke, 42, 3291-3293. http://dx.doi.org/10.1161/STROKEAHA.111.625046 
Scientific Research Publishing (SCIRP) is one of the largest Open Access journal publishers. It is currently publishing more than 200 open access, online, peer-reviewed journals covering a wide range of academic disciplines. SCIRP serves the worldwide academic communities and contributes to the progress and application of science with its publication.

Other selected journals from SCIRP are listed as below. Submit your manuscript to us via either submit@scirp.org or Online Submission Portal.
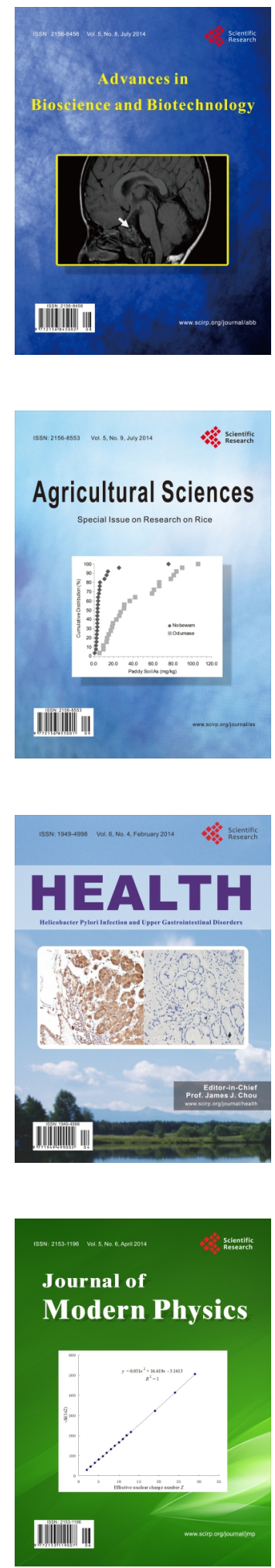
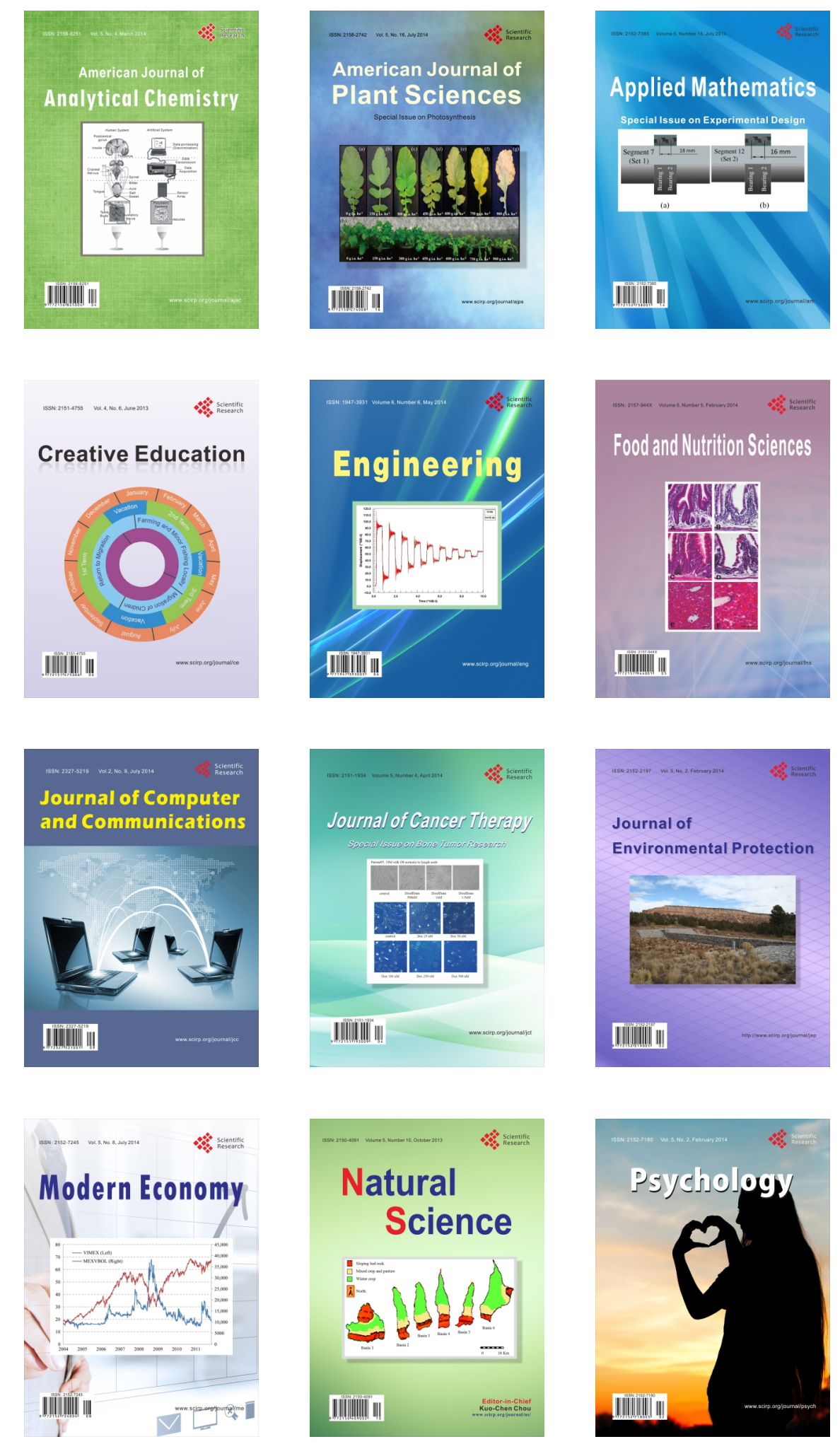